\title{
Visualization of Protein-Lipid Interactions in Connexin-46/50 Intercellular Communication Channels at $2.1 \AA$ Resolution
}

Jonathan A. Flores ${ }^{1,2}$, Kimberly A. Dolan ${ }^{1}$, Bassam G. Haddad ${ }^{1}$, Janette B. Myers ${ }^{1}$, Craig C. Yoshioka ${ }^{3}$, Daniel M. Zuckerman ${ }^{3}$, Steve L. Reichow ${ }^{1, *}$

1. Department of Chemistry, Portland State University, Portland, OR, USA.

2. Department of Biochemistry and Molecular Biology, Oregon Health and Science University, Portland, OR, USA.

3. Department of Biomedical Engineering, Oregon Health and Science University, Portland, OR, USA.

* Corresponding author: reichow@pdx.edu

One of the most direct ways to transfer chemical information between cells is through the use of a specialized family of membrane channels, known as gap junctions. Multicellular organisms depend on gap junctions to coordinate metabolic activity, provide pathways for electrical coupling and buffer against chemical insult. These functions are facilitated by a unique macromolecular architecture. In each cell, a "hemi-channel" is formed by the oligomerization of six individual subunits (called connexins). Each hemi-channel forms a barrel-like structure, and the fusing of two hemi-channels from opposing cells results in the formation of a complete dodecameric channel that effectively couples the cytoplasm of the two connected cells. Once formed, these intercellular channels establish a continuous aqueous pathway for coupled cells to transfer chemical information (i.e., communicate). An enormous pore of $\sim 1.5 \mathrm{~nm}$ facilitates direct passage of water and ions (e.g., $\mathrm{Na}^{+}, \mathrm{K}^{+}$and $\mathrm{Ca}^{2+}$ ), but also small signaling molecules such as ATP, cAMP and $\mathrm{IP}_{3}$, hormones, nutrients and even small molecule drugs. In this way, individual cells may communicate across an entire tissue or organ and coordinate long-range signaling events in nearly every tissue in our body.

Humans express 21 connexin isoforms throughout the body, in a tissue-specific fashion. Our laboratory recently characterized gap junction intercellular channels isolated from native lens tissue, formed by connexin-46/50, using single particle CryoEM [1]. The structure was shown to adopt a stabilized openstate conformation, as compared to the structure of connexin-26 previously determined by X-ray crystallography [2]. Here, we used lipid nanodisc technology to incorporate Cx46/50 intercellular channels into a more native-like environment, closely mimicking the cell-to-cell junction. Structural characterization of Cx46/50 lipid-embedded channels by single particle CryoEM revealed a drastic lipidinduced stabilization to the channel architecture, resulting in a $3 \mathrm{D}$ reconstruction at $2.1 \AA$ resolution providing an unprecedented level of detail for the class of protein [1-3], and in a near-native lipid environment. The subunit packing within each hemi-channel is buttressed by a bouquet of highlystabilized lipids. Remarkably, the stabilizing effects to the lipid-bilayer extend well beyond the first layer of annular lipids and effectively immobilizes the extra-cellular leaflets of the two connected membranes. In addition, over 150 water molecules are resolved within the channel architecture. Ordered waters contribute to the interaction network that defines the intercellular permeation pathway, and are integrated into the core of the trans-membrane domain of the protein. Lipid and water binding sites identified in Cx46/50 are remarkably conserved across the connexin family, suggesting these stabilizing features are key contributors to the structure and function of gap junction intercellular communication. The functional relevance of lipid-binding sites in Cx46/50 will be discussed in the context of the native environment of the eye lens. 


\section{References:}

[1] Myers, J.B. et al., Nature 564 (2018), p. 372.

[2] Maeda, S. et al., Nature 458 (2009), p. 597.

[3] Benette, B.C. et al., Nat. Commun. 7 (2016), p. 8770.

[4] The authors acknowledge funding from the National Institutes of Health, NIGMS (R35-GM124779) to SRL, NEI (1F31EY030409-01) to JAF, and BUILD EXITO program (NIH common fund:

UL1GM118964) to KD and JAF. JAF and KD contributed equally. 